brane should read soft tissues, meaning periodontal and mucous membrane.

I was led to the above statement from the fact that many instances were found (about 4 per cent.) where the apices of the teeth perforated the bony floor of the antrum, having no bony covering, and by close examination it seems clear to me that this condition obtained during life, especially since one case was found among the recent specimens of the Army Medical Museum when the skulls and recent specimens were being examined.

Within the past few weeks I have examined twenty more recent subjects on this point as well as others, and found a case where nothing but soft tissue covered the apex of a buccal root of a first molar. These two cases show that such a condition does obtain during life, and furnish a good foundation for the belief, that when skulls are found with no bony covering to the apices of the teeth, that during life they probably had no other covering than soft tissue.

Since writing the paper referred to, three cases of empyema of the maxillary sinus have come under my observation, none of which were in any way traceable to disorders of the teeth, but in each one the exciting cause was intranasal; thus adding to the evidence that the trouble is due in the larger number of cases to intranasal disorders, as claimed in former papers.

Illuminating these cavities by the mouth lamp, is a most valuable adjunct to diagnostic symptoms; the three cases referred to, all threw the diagnostic shadow.

\section{THE TECHNIQUE OF RESECTIONS OF THE SKULL.}

BY ALLEN DEVILBISS, M.D. TOLEDO, OHIO.

Surgical work should not be undertaken until the nature and location of the disease is first determined. In a limited number of cases it may be advisable to remove portions of the cranial bone or bones for explorative purposes. Localization of cerebral functions has made it possible in certain cases to make an accurate diagnosis. There are large portions of the cortex, that the functions of are not understood, but the sensor motor area, the visual area, the speech area, the auditory area, and the areas of sensations of smell and taste are known, and these facts in connection with the various symptoms will generally guide to a correct idea of the nature and situation of the lesion.

Trephining may be performed for the removal of foreign substances, spicula of bone, depressed bone, blood clot, the opening of an abscess, the removal of cysts, excision of tumors, for the relief of intracranial pressure, for drainage, etc. Any one of the conditions just mentioned may be the cause of epilepsy, insanity, paralysis, or most any of the brain lesions with which we have to contend.

While I do think that we should not hasten to operate in all cases, yet we must remember there is danger in delay. Delay of surgical interference in recent fractures has often resulted in death or brought about conditions that make death preferable to life. We should not hesitate to open the mastoid cells when there exists an abscess. If done early, it is sure to prevent further mischief, but if left alone quite a proportion go on to infection of the lateral sinus, meningitis or abscess of the brain. The suc- cess of cerebral operation depends upon every detail of aseptic surgery being carried out to perfection.

The marking off on the scalp, for the fissures of Rolando and Sylvius, is necessary in many cases and should be done before giving the anesthetic. To accomplish this, various rules have been given. Owing to the difference in shape and size of the head no land marks can be taken that are constant in their relations to the fissures, but are sufficiently so for surgical purposes. The plan that I have used is principally one of my own, and I think, it is quite definite and not complicated. I use a fiexible metallic tape for measuring, and tincture of iodin for marking. First, measure from the glabella over the top of the head to the occipital protuberance, half of the whole distance plus one-half inch back will correspond to the upper end of the fissure of Rolando; mark this point, then place one end of the flexible measure upon the point marked, and carry it along the side of the head down to the angle of the mouth. Make a mark by this line at a point, three and one-half inches from the mark on the median line. The imaginary line

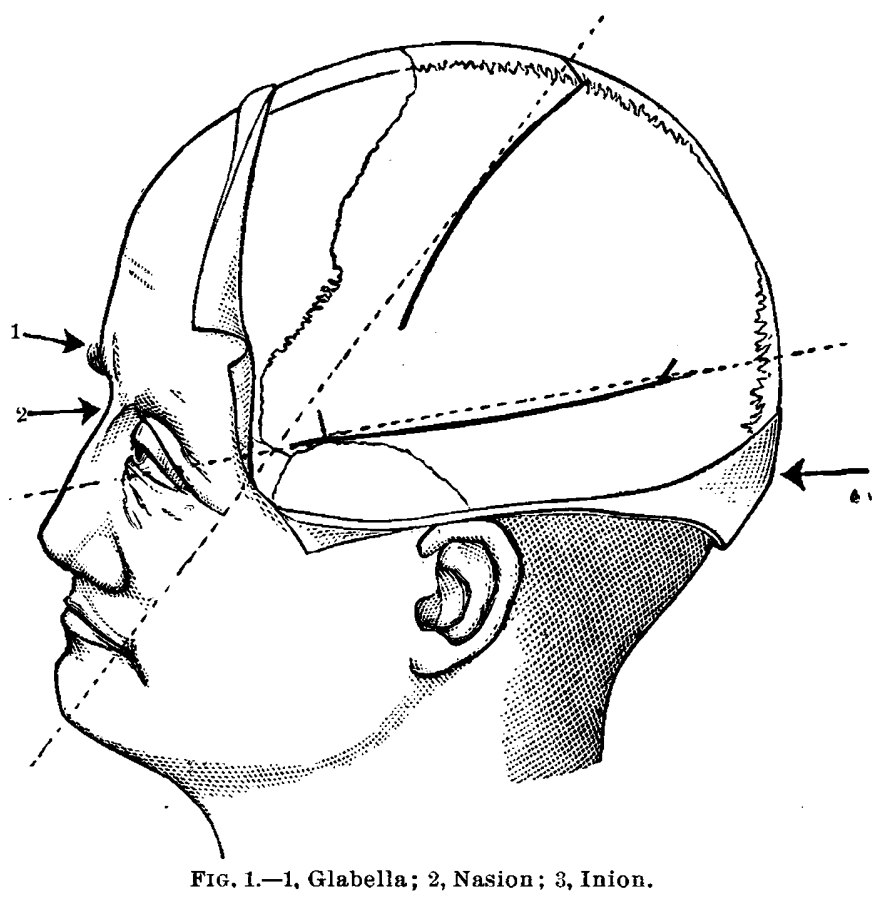

between the points marked will lie over the fissure of Rolando except at its lower portion which is more vertical. To find the fissure of Sylvius place one end of the tape at the lower end of the nasal bone, carry it along in line with the external angular process of the frontal bone to a point three-fourths of an inch below the parietal eminence; then make a mark at a point one and one-fourth inch back from the external angular process. From this point count off fourinches and mark. A line drawn from one of these points to the other will correspond to the fissure of Sylvius. The anterior limb of the fissure is about two inches behind the external angular process. By continuing the Sylvian line to the median line we form a junction at the point where lies the parieto-occipital fissure. All the areas now known can be located with a definite relation to these three fissures; hence no further rules are necessary. With the landmark made for the fissures and the patient anesthetized, we are ready to make the incision through soft parts. 
This may be done with one stroke of the knife. We stop turning as soon as the drill locks. By so doing are now ready to make the initial opening through there will be no unnecessary strain upon the cable. the bone to the dura. This can be accomplished in I also have one side-cutting probe-pointed drill that several ways. After trying the various devices used, I use wherever there would be danger in wounding I prefer to use a small-sized cone-shaped trephine, tissue by the one with the sharp end. They do the three-eighths of an inch on its face, which makes an work in less time than it can be done with the chisel opening of sufficient size to allow the easy passage of or gouge and without jarring. They are now machine the lower jaw of the forceps down to the dura. Its ground and are very accurate and will not heat if run advantages over other trephines are: 1 . Its centering at a proper speed. The harder the bone the less speed point can be held in position until placed upon the should be used. The same law that governs the speed part to be operated upon, and when it has cut deep enough for fixation, the centering point may be removed. 2. The central opening being carried twice the length of the cutting cylinder with a recess above the cutting part to allow the bone button to be shoved up through and fall out, which makes it easy to clean.

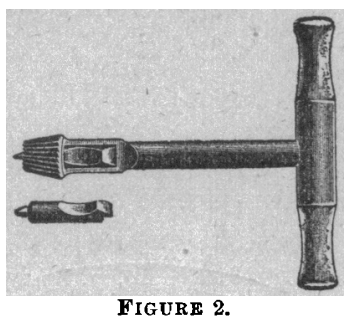

While most operations can be made by the hand trephine, the advantage of drills used by power will be readily seen, especially in cases of mastoid diseases, where there are symptoms of cerebral disease, yet the symptoms are not distinct enough to warrant

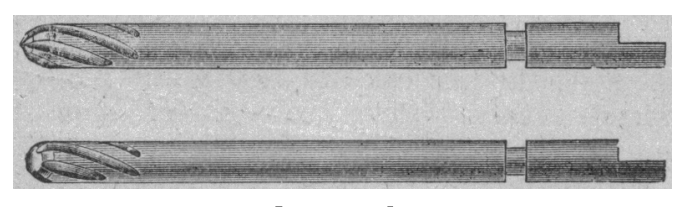

FIGs. 3 and 4.

the initial opening to be made direct, the mastoid being first opened and then extended as far as is found necessary.

I have one small drill for making explorative openings, also for enlarging them. It widens from the

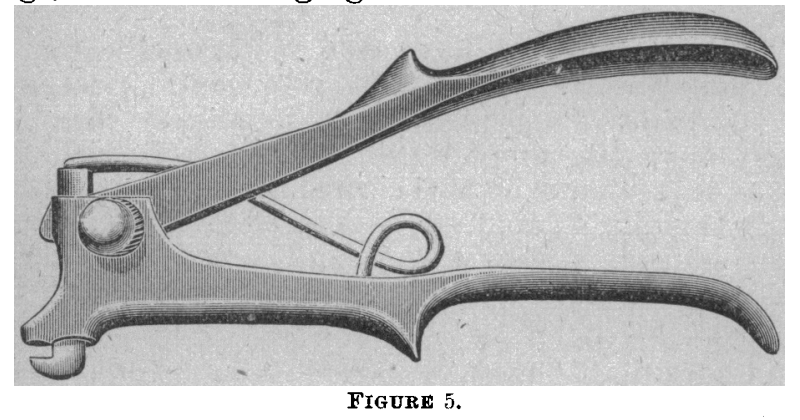

point up and has a cutting edge on its end and side. This causes it to lock and stop when the point is ready to pass through the bone; there being no more bone to cut with the point, the side of the drill is pressed into the wall of the hole. This stops its revolving just when the hole is completed, or when there is only a thin sheet of bone not thicker than writing paper left, which may be pushed out with a probe-pointed drill, or cut out with a stop drill by holding it back so that it can cut but little at each revolution to prevent its locking. In this way, the first opening is made without danger of wounding the tissue beneath the bone. When using this drill it is best to instruct the person furnishing the power to in drilling metal applies to the drilling of bone. When the initial opening is made directly through the skull bone to the dura and I wish to enlarge it, or cut a slot out or make a trap-door, I use a bone-cutting forceps that I have devised for this purpose. It will cut a slot in any direction desired. The lower jaw shoves the piece cut off out through the upper jaw, leaving the edge of the bone smooth next the dura. The under-surface of the lower jaw being round and smooth serves as a dural separator. The leverage is such that there is no difficulty in biting through. After the slot is two inches long, it is best to take

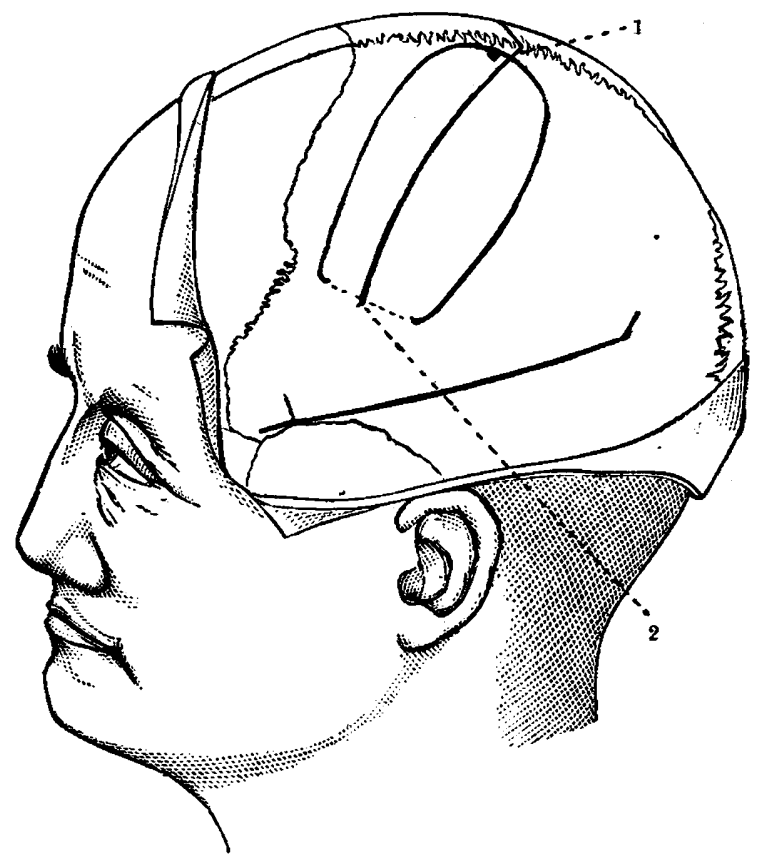

FrG. 6 , 1, Point of initial opening : 2, Fracture lime. Forceps should be turned at each end of fracture line as shown.

side bites so as to make an opening large enough for the lower jaw to pass through and not go back to the place of beginning. It can be made aseptic. as its parts separate by removing the thumb screw. I use a jaw that cuts one-eighth of an inch. Some prefer one only one-sixteenth inch so as to have bony union instead of fibrous.

The lower jaw is made so that it does not fit closely the opening in the upper one. By having a little space left it clears itself better and yet leaves the bone smooth next the dura. There may be slight nicking of the extermal surface of the bone, which is imnaterial. I have used the lower jaw flat upon its face and also deeply cupped. I find that this makes but little difference in regard to the smoothness of the surface or the ease with which it is accomplished. I have used it many times and I find no bleeding from the diploe. It is prevented on account of the way it shoves the bone out. When making a trapdoor brain operation of sufficient size to cover motor 
areas, situated along the fissure of Rolando, say two by three inches, I have adopted the following plan: Make an opening at the center of the upper end of the portion to be turned over; then take the forceps and bite out piece after piece until the point to be fractured across is reached; then turn the forceps in the direction of the line to be fractured and take a bite or two; next cut out from the central opening to a point where the other end of the fractured line is to be, take a side bite or two in the same manner as done upon the other side. This leaves the part to be fractured across weaker than at any other point and can be easily broken. Next take a vectus and lift or pry it up and break it over. After completing the work necessary within, the flap may be returned. The fractured end may be placed even (or up, if need be, to increase intracranial room), and the rough surface of the fractured parts will hold it in place, if pressure be maintained until it is put in proper position. The semi-circular or horse-shoe-shaped bone flap with soft tissues attached and continuous with the scalp at its base which contains the blood supply for the parts,

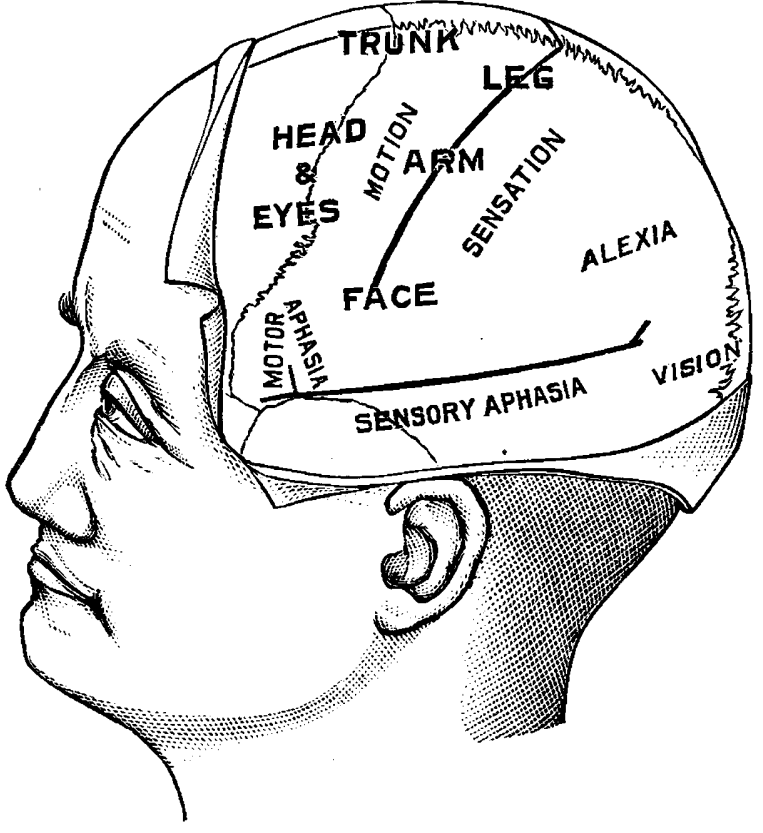

Figure 7.

are to be preferred to any other procedure except in recent fractures; no extension of the incision should be made in the direction of the line of the fracture, nor is it necessary to do any chiseling or cutting of any kind of the bone along the line to be broken. The connection between the soft parts and bone should be disturbed as little as possible.

For operation upon flat bones there is no other instrument equal to the forceps that I have described to you, whether it be by skilled or unskilled hands. In unskilled hands it is certainly safer than the chisel and mallet, for the reason that when the instrument is placed in position to bite, it shoves the dura out of tho operative field, and by its use the injurious effects are prevented that may occur from the blows when the chisel and mallet are used.

When using it I prefer to take the lower handle in one hand, the upper one in the other, so that I can carry the bit up or down at will, and place firmly its stem against the bone, in this way a full bite can be taken out each time. A large trap door can be made in from five to ten minutes. That there are more permanent cures made by this plan of operating I have descriled, I can not say, but I can say that death and other bad results from shock incident to the prolonged use of the mallet and chisel may be prevented.

Many surgeons have used it in making craniec. tomies and proved its usefulness; but this is not its only field, it extends to thoracoplasty and laminectomy and all bones that are thin enough to come within its jaws.

When in Chicago recently I found this instrument in use in several of the schools.

\section{CATAPHORESIS.}

Read in the Section on Dental and Oral Surgery, at the Forty-seventh Annual Meting of the American Medical Association, beld at Atlania, Ga., May 5-8, 189\%.

BY HENRY W. GILLETT, D.M.D.

NEW PORT, R. I.

The primary facts upon which the present practice of cataphoresis are based were first discovered, nearly forty years ago, by Dr. B. W. Richardson. The development of practical results from these facts has been slow, but the past five or six years have added much to the general knowledge and application of them.

The fact that the galvanic current does, under proper conditions, carry with it into the tissues of the body, fiuids and the substances they may hold in solution, is now sufficiently well known and accepted. The practical realization and use of this fact has not been general for numerous reasons, but now that the principles of the process are becoming more widely understood, it seems quite probable that some of the most important applications of this method of medication will be in the treatment of the oral tissues.

Thedentine of the human tooth, which, for generations, we have been wont to consider practically impenetrable by drugs, has, by reason of our better understanding of the process of cataphoresis, changed its relative position, and may now be considered one of the tissues easily penetrated and influenced by drugs.

The very fact that has made it impossible for us to cause absorption of drugs into dentine, namely, its comparative non-vascularity, is an aid when we apply them by cataphoresis. Into a very vascular tissue it is difficult to feed a cocain solution with sufficient rapidity to produce a deep effect, because the blood current washes it away and spreads it through the system. In dentine this does not occur.

There are material differences necessary in the apparatus to be used with success for treating sensitive dentine, and that which may be used for other tissues. For some subjects almost any voltage of current may be used if it is choked down in quantity; but a large percentage of the subjects who most need the aid of this method will be found too sensitive to the effect of the galvanic current to allow of its use on sensitive dentine, unless the apparatus is so arranged that the voltage may be reduced to a very low point at the start, and gradually increased, in small steps, to a point where it will be efficient in producing cataphoric effects in a reasonable time. McGraw used the ordinary battery apparatus in 1888 for treatment of dentine, but gave it up because of crudeness of apparatus. With such apparatus it is necessary to either commence with the full voltage, or to add it in 